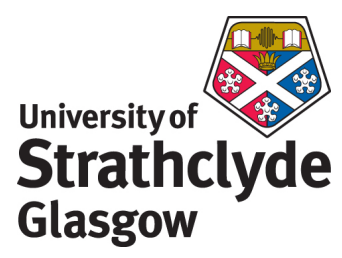

Brown, R.E. and Phillips, C. and Kim, H.W. (2010) Helicopter brownout - can it be modelled? In: Royal Aeronautical Society Rotorcraft Group Conference: Operating Helicopters Safely in a Degraded Visual Environment, 16-17 June 2010, London, UK.

http://strathprints.strath.ac.uk/27364/

Strathprints is designed to allow users to access the research output of the University of Strathclyde. Copyright $(C$ and Moral Rights for the papers on this site are retained by the individual authors and/or other copyright owners. You may not engage in further distribution of the material for any profitmaking activities or any commercial gain. You may freely distribute both the url (http://strathprints.strath.ac.uk) and the content of this paper for research or study, educational, or not-for-profit purposes without prior permission or charge. You may freely distribute the url (http://strathprints.strath.ac.uk) of the Strathprints website.

Any correspondence concerning this service should be sent to The Strathprints Administrator: eprints@cis.strath.ac.uk 


\title{
Helicopter Brownout - Can it be Modelled?
}

\author{
Catriona Phillips* Hyo Won Kim ${ }^{\dagger} \quad$ Richard E. Brown ${ }^{\ddagger \S}$ \\ Rotorcraft Aeromechanics Laboratory, Department of Aerospace Engineering \\ University of Glasgow, Glasgow G12 8QQ \\ United Kingdom
}

\begin{abstract}
Significant progress has been made to date in modelling, computationally, the formation and development of the dust cloud that forms in the air surrounding the rotorcraft under brownout conditions. Modern computational methods are able to replicate not only the development of the dust cloud in appropriate operational scenarios, but also the sensitivity of the shape and density of the dust cloud to the detailed design of the rotorcraft. Results so far suggest that attempts to ameliorate brownout by aerodynamic means, for instance by modifying the rotor properties, will be frustrated to some extent by the inherent instability of the flow field that is produced by the helicopter. Nonetheless, very recent advances in understanding the fundamental mechanisms that lead to the formation of the dust cloud may allow substantial progress to be made once certain elements of the basic physics of the problem are more fully understood and better quantified.
\end{abstract}

\section{Introduction}

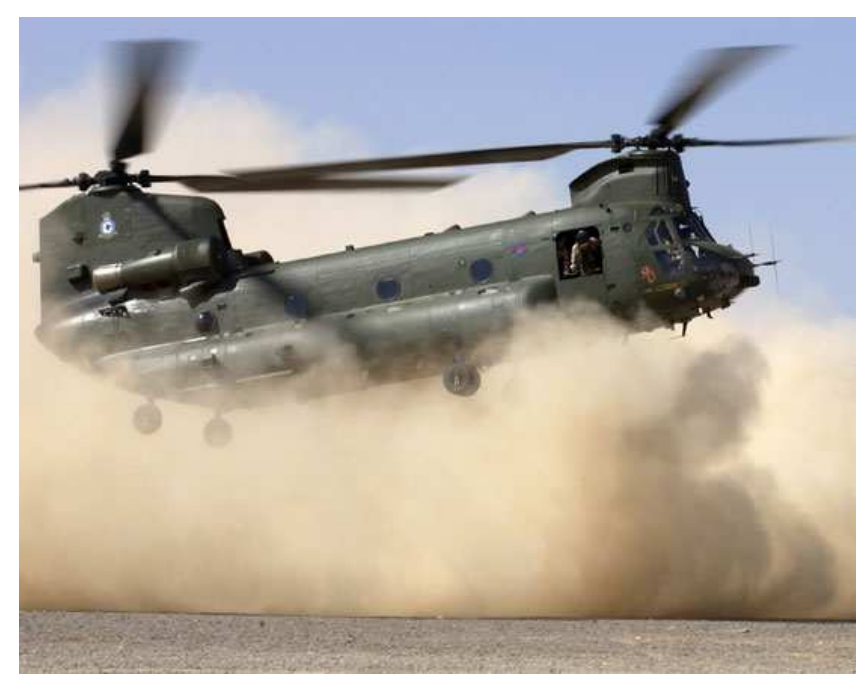

Figure 1: Chinook helicopter landing in dusty conditions. Note the cloud of dust that is being produced below the aircraft; brownout is a distinct possibility should the aircraft decelerate or descend. (Image Crown copyright.)

\footnotetext{
* Postgraduate Research Assistant.

$\dagger$ Postdoctoral Research Assistant.

$\ddagger$ Mechan Chair of Engineering.

$\S$ Corresponding author; e-mail: rbrown@aero.gla.ac.uk
}

Presented at the Royal Aeronautical Society Rotorcraft Group Conference, "Operating Helicopters Safely in a Degraded Visual Environment," London, June 16-17, 2010. Copyright (C)2010 by the authors. All rights reserved.
A particular problem to helicopter operators in desert or dusty conditions is the possibility of entrainment of dust from the ground into the air when the vehicle is operated close to the ground. This process can cause large clouds of dust to form in the air surrounding the helicopter. The possibility exists, under certain operational conditions, that these clouds might obscure the pilots' view, or provide them with false cues as to their motion relative to the ground, leading to a loss of situational awareness in a condition known as 'brownout' (see Fig. 1).

Two routes, both potentially leading to the mitigation of brownout in practice, have been proposed. The first route is to alter the geometry or operating conditions of the helicopter. If the aerodynamics of the vehicle can be suitably modified as a result, then the development of the brownout cloud might be changed in a manner that is more conducive to the piloting task. The second, somewhat more pessimistic (some might say pragmatic) avenue is to employ an onboard system to augment the pilots' conventional visual cues - for instance using sensors or electronically-generated imagery. A pre-requisite to both approaches is a detailed understanding, from basic physical principles, of the evolution of the dust cloud - in the first instance since aerodynamic amelioration of brownout is essentially dependent on such an understanding, and, in the second instance, to inform the specification of such devices and to optimise their placement on the airframe. 
Thus, significant effort in a number of university laboratories and research establishments around the world is being expended to try to understand the fundamental physics that underpins the development of the brownout cloud. Some significant progress has been made to date in understanding the phenomenon and in producing computational models that are able to replicate, not only the development of the dust cloud in appropriate operational scenarios, but also the sensitivity of the shape and density of the dust cloud to the detailed design of the rotorcraft (Fig. 2).

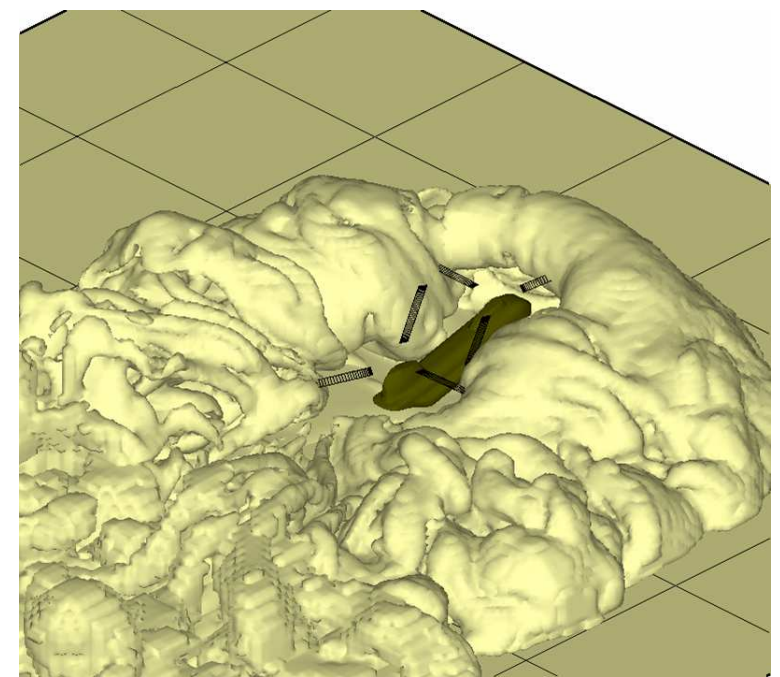

Figure 2: VTM/PTM Simulation of the dust cloud that is formed in the air surrounding a generic tandem helicopter during its approach to a desert landing (Ref. 1).

In this paper, some of the progress to date is summarised. It will be argued that brownout requires highly-sophisticated, specialised computational models to resolve accurately the development of the brownout cloud. When these models are used, the physics of brownout is found to bear a highly complex and non-linear relationship to the detailed design of the rotorcraft. Results so far suggest that modification of the rotor properties to ameliorate brownout by aerodynamic means will be a difficult, but by no means an impossible, task. Indeed it is hoped that the reader will infer from what is written here that the modern generation of computational models, when properly used and interpreted, are capable of yielding deep insights which can be translated into an objective assessment of the feasibility of almost all proposed ameliorative strategies in their real-world, operational context.

\section{Empirical Data}

All modelling efforts need to be informed and guided by reference to measurements taken in the real world. Full-scale experimental data are rare, however, and what data there are, are bedevilled by the usual problems of data quality and repeatability that are characteristic of full-scale flight testing. Available data include Rodgers's somewhat technologically-constrained 1968 measurements of the particulate distribution near the fuselage of a Piasecki H-21 tandem-rotor helicopter in hover above the desert surface (Ref. 2), and, although somewhat less readily accessible to researchers outside the USA, the data produced during the more recent 'Sandblaster' series of tests conducted by the U.S. Army in 2007 at the Yuma Proving Grounds in Arizona (Ref. 3).

Laboratory-scale experiments on subscale rotor systems offer the possibility of obtaining somewhat better-conditioned data under controlled conditions. Notable in this respect are the experiments by Green at the University of Glasgow (Ref. 4) and by Leishman's group at the University of Maryland (Ref. 5). For the very small rotors used in these experiments, however, it is a notoriously difficult task to match the physical properties of the particulates used to represent the dynamics of the dust cloud in the laboratory with those of the particles known to be responsible for the brownout cloud in full-scale practice. Despite these scaling issues, these experiments have provided, at least, very good qualitative insight into some of the physical processes that are at work during the development of the brownout cloud. They have also aided the development of a range of computational models that aim to capture, from first principles, the details of the formation and development of the brownout cloud.

\section{Computer Modelling}

Two rather different approaches are currently used to model the evolution of the particulate matter as it is entrained from the ground into the flow, and subsequently convected into the air around the helicopter to form the dust cloud that is responsible for brownout. The first, so-called Lagrangian approach, favoured by researchers such as Leishman and co-workers at the University of Maryland (Ref. 6), Wachspress et al. at Continuum Dynamics Inc. (Refs. 7 and 8), and D'Andrea at AgustaWestland (Ref. 9), models the distribution of particulates in the flow via the dynamics of a set of surrogate, or 'marker' particles. The motion of these particles in response to the drag force exerted on them by the flow is determined by integrating, numerically, Newton's second law of motion.

The major advantage of the approach is that it can, at least in principle, model quite straightforwardly the dynamics of a distribution of particles with widelyvarying properties, as is characteristic of the desert surface. This major advantage will allow Lagrangian methods to come into their own particularly if the detailed mechanisms whereby sediment is entrained from the ground into the air are ultimately to be resolved 
from first principles. The community is very likely to be forced in this direction by the current controversy, as alluded to later, regarding the appropriateness, particularly in the helicopter context, of the current theoretical basis that is used to provide this crucial link in the causal chain that leads to brownout conditions in practice.

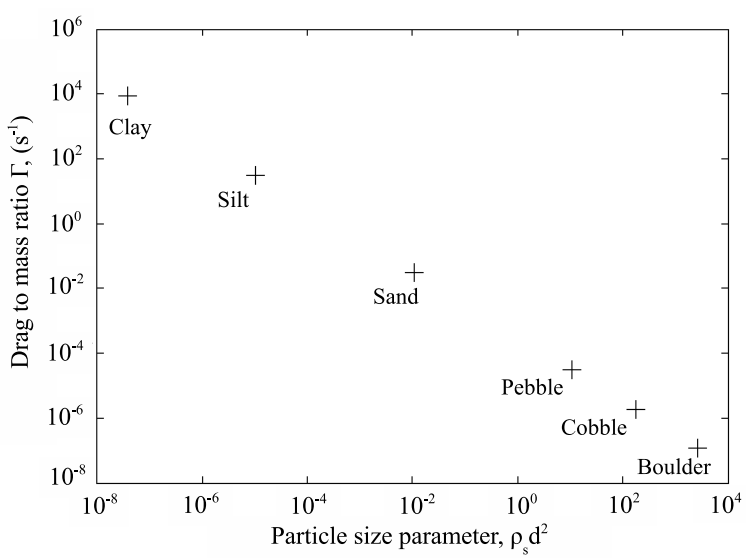

Figure 3: Particle drag to mass ratio of the most common constituents of the desert surface (Ref. 10).

As shall be argued shortly, however, the particles that are primarily responsible for brownout are very close to being in equilibrium with the forces acting upon them. This yields a so-called 'stiff' integration problem when using the Lagrangian approach, with the result that usually a large numerical error in integrating the trajectories of the marker particles must be accepted. The alternative is to run the simulations at a timestep that can be uneconomical given the large number of individual particles that must be simulated. Lagrangian calculations lose accuracy very quickly when the distribution of marker particles becomes diffuse in any region of the flow, and this is a particular problem when the distance between marker particles becomes comparable to the scale of the structures that are present in the flow. Unfortunately, the flows that are produced by helicopters are characterised by the existence of structures with length-scales that range from being comparable to the rotor diameter down to less than one hundredth of that size. Thus Lagrangian models tend to become inefficient and somewhat unreliable, even misleading in the worst instances, when modelling the details of the evolution of the dust cloud in the air surrounding the helicopter. For this reason the authors of this paper prefer an alternate formulation of the problem (Refs. 1, 10 and 11).

In this alternative, so-called Eulerian approach, the particulate load in the flow around the helicopter is represented via the density of the particles within the flow rather than by the positions of the particles themselves. The same statistical mechanics formalism that can be used to derive the equations of fluid dynamics from their fundamental molecular basis can then be employed to derive a partial differential equation that governs how the dust density distribution $\rho$ evolves within the flow that is produced by the helicopter:

$$
\frac{\partial}{\partial t} \rho+\left(v+v_{g}\right) \cdot \nabla \rho=S+\delta_{s} \nabla^{2} \rho+H(\rho)
$$

In this equation, called here the Particulate Transport Equation (PTE for short), $v$ is the flow velocity, $v_{g}$ is the fallout velocity of the particles due to gravity, $\delta_{s}$ is a diffusion coefficient, and the source term $S$ is only non-zero in a thin layer just above the ground. $H(\rho)$ is a set of residual differential terms that become significant if the statistics of the particle distribution are perturbed far from equilibrium - a situation to be explored in more detail shortly. The PTE expresses in mathematical form the fact that the particulate density at any point in the flow around the helicopter originates from the ground, and evolves in time by the dual processes of convection by the fluid and diffusion as a result of the random motion of the particles relative to each other. Finally, gravity acts to remove particulates from the flow and to return them to the ground at a finite rate. The major advantage of the Eulerian approach is its mathematical rigour, and hence the confidence that can be placed in its predictions in the absence of direct corroborative data. Indeed, relatively straightforward numerical algorithms can be devised to solve the equations of motion of the particulate distribution within the brownout cloud when cast in the form of the PTE, and these algorithms can be shown formally not to suffer inherently from the problems of spatial and temporal accuracy that are associated with their Lagrangian cousins. In addition, the neces-

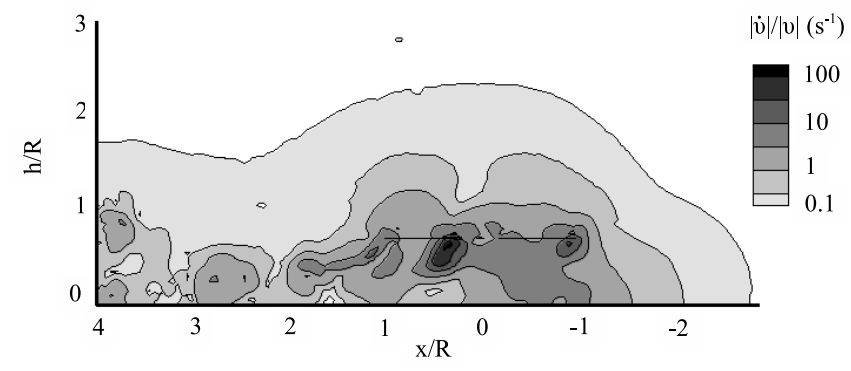

Figure 4: Relative acceleration parameter on a longitudinal slice through the flow surrounding a Blackhawk-class helicopter in low-speed forward flight above the ground (Ref. 10). The helicopter is flying from left to right and the rotor position is marked by the horizontal black bars.

sity for the additional terms $H(\rho)$ on the right-hand side of the equation (or, in other words, the validity of the model without those terms) can be determined rigorously by comparing the relative magnitude of two parameters that can be measured directly within the flow. The model becomes increasingly valid when the 
drag to mass ratio of the particles taking part in the process, $\Gamma=18\left(\rho_{a} / \rho_{s}\right)\left(\delta / d^{2}\right)$, where $\rho_{a}$ is the air density and $\rho_{s}$ the particle material density, $d$ the particle diameter and $\delta$ the viscosity of the air, is large compared to the local relative acceleration $|\dot{v} / v|$ within the flow. Importantly, the relative acceleration is a property solely of the helicopter system being studied.

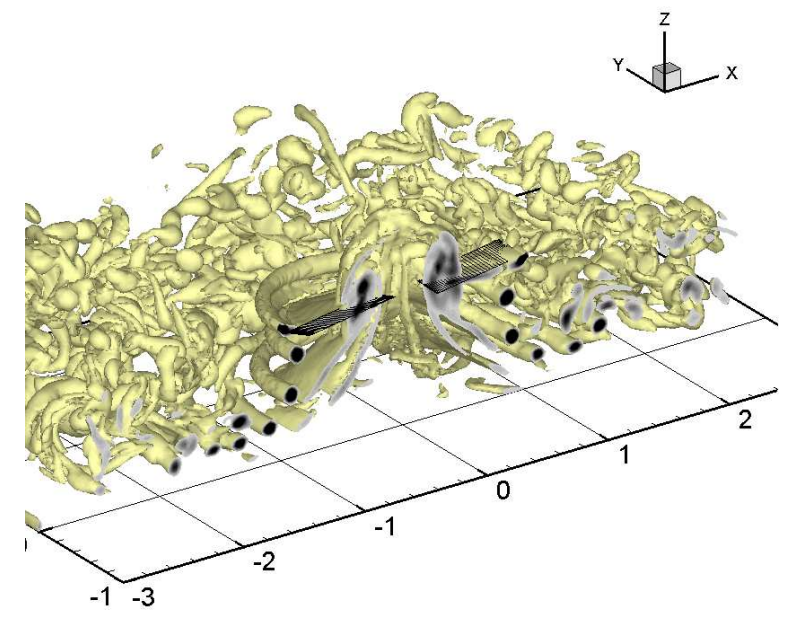

(a) Vorticity distribution

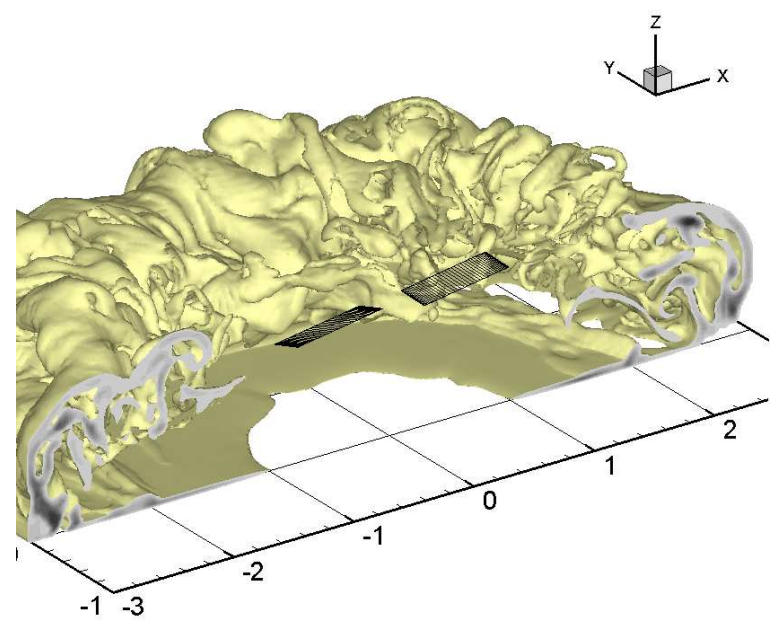

(b) Dust distribution

Figure 5: Vorticity distribution (a) and dust distribution (b) in the flow field below a two bladed rotor in hover above the ground as predicted by the VTM/PTM. (Blade positions represented as hatched surfaces, flow sectioned through the rotor centreline.)

As an example, Fig. 3 shows the range of drag to mass ratios that is typical of the particles encountered on the desert floor. Figure 4 shows contours of the computed local relative acceleration within the flow surrounding a Blackhawk-class helicopter in slow forward flight at just over half a rotor radius above the ground. It is known that it is the finest dust particles that are responsible for the prime characteristics of the brownout cloud (the larger particles, especially sand and grit, are of course also relevant to helicopter operations, being primarily responsible for the erosion of rotor blades and turbomachinery). The separation of at least two orders of magnitude between $\Gamma$ for these finest particles and the relative acceleration throughout the majority of the flow shows that the PTE without the extra terms $H(\rho)$ on the right-hand side is entirely adequate for the prediction of the dynamics of the brownout cloud that is produced by the fullscale system - at least for all engineering purposes. Also, by inference, the analysis shows that the particles comprising the brownout cloud are in near-equilibrium with the forces acting upon them throughout the majority of the flow field surrounding the helicopter.

The relative acceleration within the flow scales according to the rotor speed, however. If the flow shown in Fig. 4 is re-scaled to be appropriate to a micro-rotor with operating parameters similar to the rotors used to generate the published experimental data referred to earlier, then the relative acceleration parameter turns out to be about an order of magnitude higher than for the full-scale system. The same dust particles cannot thus be expected to behave in the same way in the experimental system as in the full-scale system, reinforcing the argument regarding the direct relevance of subscale experiments to full-scale practice alluded to earlier. Add to this the observation that all modelscale experiments to date seem to have used particulates with a mass to drag ratio as much as two orders of magnitude greater than the particulate matter that forms the principal constituent of the brownout cloud in the full-scale situation, and it is the authors' opinion that these tests, as dramatic and visually-appealing as they are, are in danger of leading the community to a possibly misleading impression of the importance of non-equilibrium effects on the particle dynamics and possibly also to an over-emphasis of the importance of re-entrant material on the entrainment of particulate matter from the ground into the air. These issues can and will be addressed, but, for the moment at least, it appears that numerical methods offer the only viable approach to accessing and understanding the detailed physics that underpins the development and formation of the full-scale brownout cloud.

Both the Lagrangian and the Eulerian approaches are hostage however to the accuracy of the particular models that are used to represent the manner in which the particulates leave the ground surface and enter the flow (in other words, to the way that the source term $S$ is represented in the PTE). Much has been written, and a number of vastly complicated models, containing large numbers of tuneable parameters, have been described in the literature. This approach somewhat misses the point and runs the risk of heading up an overly academic dead end. Certainly any results built on such a shaky foundation of interconnected submodels will lack the generality required to address the 
brownout problem from a robust engineering perspective. For the moment the most pragmatic approach consistent with Occam's razor seems to be to borrow from the sedimentology community where many years of experience has gone into developing various models for the entrainment of particulate matter from river beds and dunes into the flow above. The most widelyaccepted approach in that community holds that particles do not leave the ground and enter the flow unless the flow velocity just above the ground (a concept that admittedly needs to be placed on a more rigorous footing) exceeds a so-called threshold velocity, $v_{t}$, that is characteristic of the composition of the particular surface in question. Paring down to basics, these models can all be expressed in general form as

$$
S=\kappa \Phi\left(\max \left(1, v / v_{t}\right)-1\right)
$$

where $\Phi$ is an algebraic function and $\kappa$, although unavoidably dependent on the surface properties, can be treated as one of a manageable number of parameters of the simulation - in the same vein as the helicopter weight and so on. As our knowledge advances this model will no doubt need to be augmented. A safe bet is that the first addition that will prove necessary to the model will be a differential term that represents the dependence of particulate entrainment on the rate of change of the flow velocity just above the ground surface. This term will be needed to account for the fact that, unlike in the flow away from the ground, the particle dynamics just above the ground surface (where the behaviour of much larger particles are relevant to the physics than elsewhere in the system) is forced well away from equilibrium by the action of the rapid temporal variations in the flow that are induced by the passage, close to the ground plane, of strong, compact vortical structures that exist within the wake of the rotor. The physics of sediment entrainment from the ground plane in the specific helicopter context is no doubt the area which could most benefit from further research and, indeed, where a breakthrough in understanding could contribute most to improving our confidence in the ability of predictive methods to capture the detailed mechanisms at the origin of the brownout cloud. In this vein, some very interesting experiments have already been performed, albeit in unrelated context, most notably by Dalziel's research group at Cambridge University (Ref. 12).

\section{Hierarchy of Models}

The PTE in its full form can be evolved in conjunction with a suitable model for the dynamics of the flow that is produced by the rotors of the helicopter to produce a very detailed description of the evolution of the brownout cloud. The results of a simulation of this type are shown in Fig. 5. Simulations of this

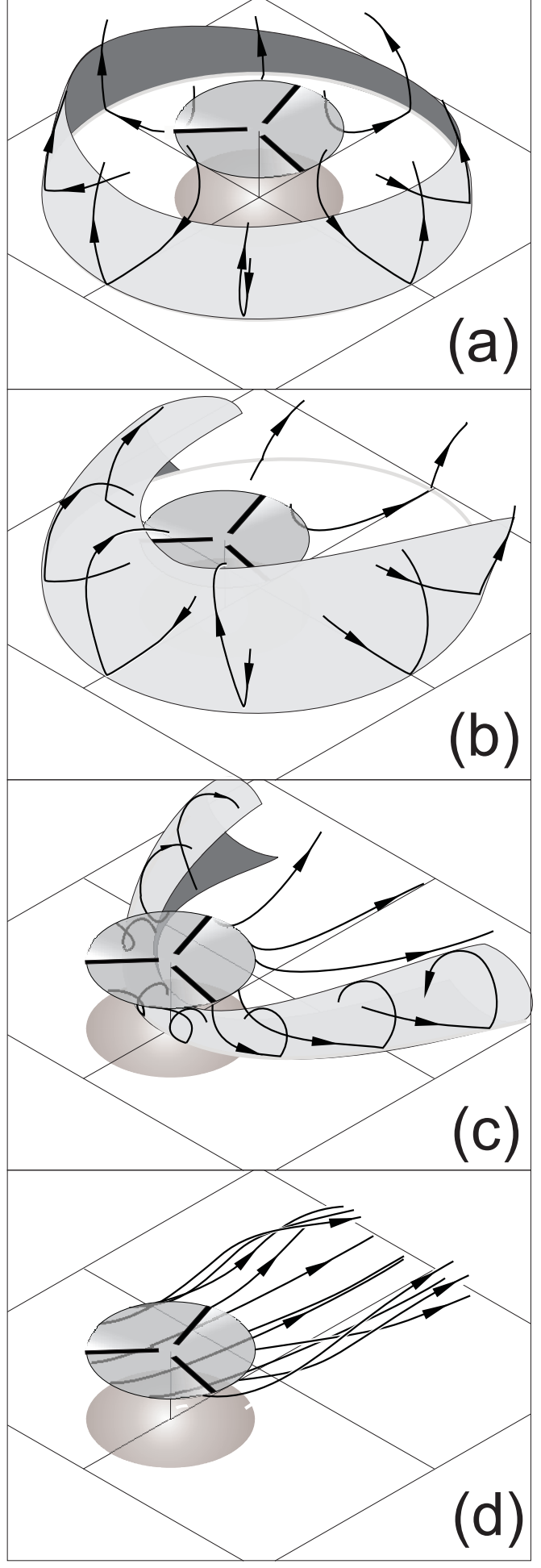

Figure 6: Flow modes and associated geometry of the brownout cloud (as represented by the shaded surface) that is produced by a rotor when operating close to the ground. (a) hover mode, (b) recirculatory mode, (c) ground vortex mode, (d) 'free air' mode.

complexity require significant computational resources to perform, however. In addition, the process of interpreting such simulations can be as opaque as the very dust clouds that are being modelled. There is thus strong motivation to find simpler representations 
of the system, particularly in order to inform the debate as to the best way forward in devising effective strategies to ameliorate the effects of brownout under operational conditions.

A very useful approximation to the dynamics of the dust cloud can be obtained by simply leaving off all differential terms on the right-hand side of the PTE, including the diffusion term, so that the evolution of the particulates within the flow is described by the simpler partial differential equation

$$
\frac{\partial}{\partial t} \rho+\left(v+v_{g}\right) \cdot \nabla \rho=S
$$

The solution to this equation is then that the dust density distribution simply follows the trajectories of the velocity field $v+v_{g}$ after originating on the ground surface. To the casual observer, the dust cloud appears as an amorphous, swirling mass of material, but careful observation shows the cloud to possess definite, although highly transient, structure (see Fig. 5 for instance, which reveals the existence of both sheet-like and filament-like structures within the dust cloud). As shown later in this paper, this simplified model is capable of explaining the existence of this structure. At this level of approximation, the velocity field that is produced by the rotor is still assumed to be unsteady and to possess structure on the smallest scales of the flow. This renders prediction of the overall shape and form of the dust cloud still a very complicated process when using this model.

A further simplification results if the velocity field that is produced by the helicopter can be decomposed as $v=\bar{v}+v^{\prime}$ where $\bar{v}$ is slowly-varying while $v^{\prime}$ is rapidly-varying and small-scale in comparison. (To be practically useful, implicit is that, although $\bar{v}$ might vary on the flight-dynamic timescales of the problem, these timescales are much longer than the local fluiddynamic timescales on which $v^{\prime}$ varies. More rigorously, this approach is useful as long as the timescale of the helicopter's manoeuvres, $T>>1 / \Omega$ where $\Omega$ is the rotational speed of the main rotor. This is nearly always the case in practice.)

If these restrictions on the velocity field are tenable, then an analysis very similar to that used to represent turbulent flows by their Reynolds-averaged properties can be employed to show that

$$
\frac{\partial}{\partial t} \rho+\left(\bar{v}+v_{g}\right) \cdot \nabla \rho=S+D(\rho)
$$

where the 'Reynolds diffusion'

$$
D(\rho)=\delta_{s} \nabla^{2} \rho+\overline{v^{\prime} \cdot \nabla \rho} .
$$

Using this approach, the gross characteristics of the dust cloud can be inferred from a relatively gross characterisation of the flow around the helicopter (for instance from the flow averaged over a number of rotor revolutions). The solution to this simplified form of the PTE is that the particulate distribution follows the trajectories of the slowly-varying vector field $\bar{v}+v_{g}$ after originating on the ground, but, unlike with the previous simplification, is additionally transported and spread across the trajectories of this field by the action of the Reynolds diffusion. At this level of approximation it should always be borne in mind that, within the flow surrounding a typical helicopter, $D(\rho)$ varies spatially over rather short length-scales and is highly anisotropic. Injudicious use of this approximate form of the PTE can thus be highly misleading.
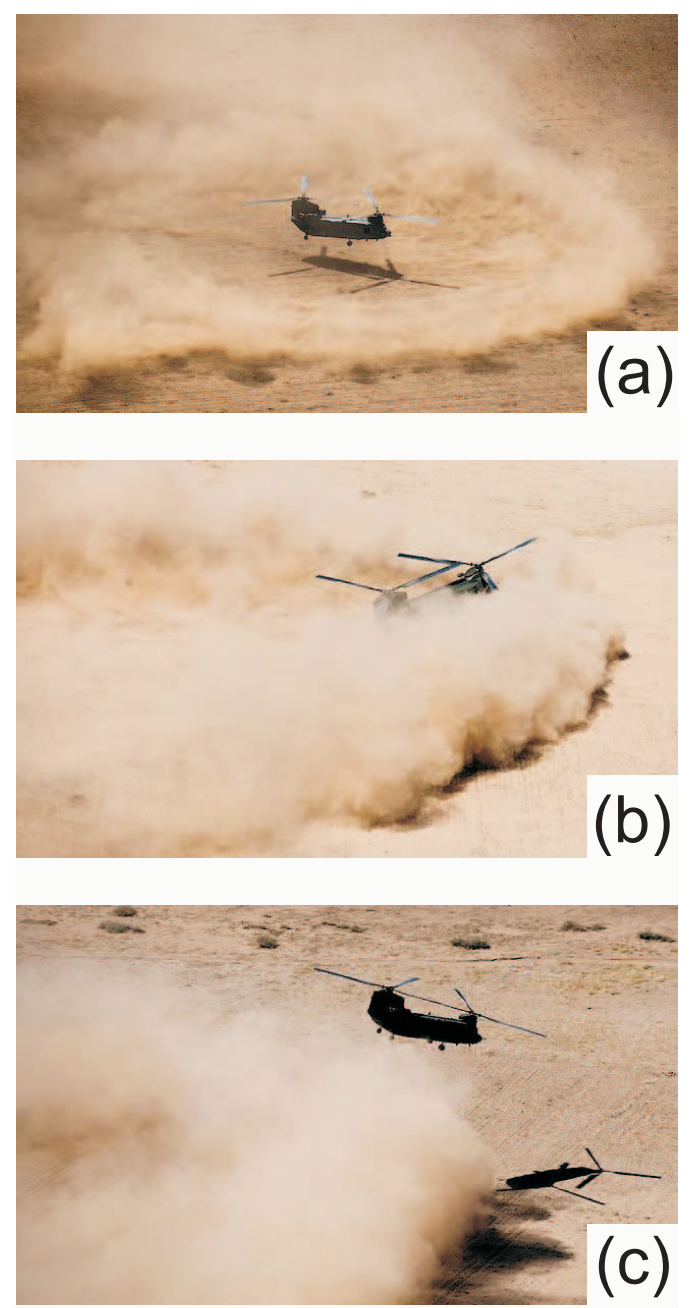

Figure 7: Geometry of the brownout cloud that is produced by a tandem-rotor helicopter when operating close to the ground: (a) hover mode, (b) recirculatory mode, (c) ground vortex mode. (All individual images Crown copyright.)

\section{Rotors in Ground Effect}

The analysis presented above implies that, before the dynamics of the brownout cloud can be understood, it is first necessary to understand the broad characteristics of the flow that is produced by the helicopter when operated close to the ground. 
In the process of producing lift, the rotor produces a downwash. The downwards progression of the flow through the rotor is curtailed however by the presence of the ground, and the flow is skewed rearwards as a result of any forward motion of the helicopter through the air. Curtiss et al. (Ref. 13) showed that the flow that is produced by an isolated rotor when operated close to the ground is characterised by several distinct modes depending on the speed of the rotor through the air (expressed as the thrust-normalised advance ratio) and its height above the ground (as a fraction of rotor radius).

In hover, the flow below the rotor turns parallel to the ground to form an outwards-directed radial jet, as shown schematically in Fig. 6a. As a result of the instability of the wake alluded to later in this paper, the jet does not extend out indefinitely from the rotor but stagnates, inducing the flow at large distances from the rotor to recirculate back towards the rotor.

As the forward flight speed of the rotor is increased, the flow enters the so-called recirculatory mode where the flow found in hover is distorted by the forward motion of the helicopter, causing a large vortex to form with its centre near the leading edge of the rotor. This vortex causes an appreciable portion of the flow that originates near to the ground to be entrained through the forward half of the rotor (Fig. 6b).

At higher flight speeds this vortex contracts and moves further aft to form a characteristic bow-shaped feature just above the ground (Fig. 6c). Numerical simulations suggest the existence of an intermediate, 'intermittent' mode between the recirulatory and ground vortex modes where the flow aperiodically switches from the one mode to the other (Ref. 14).

At higher flight speeds still, the ground vortex is swept downstream of the helicopter and the resultant flow structure is very similar to that created by the rotor when operating in free air (Fig. 6d).

The simplest, time-averaged transport model described earlier can then be used to explain the gross form of the brownout cloud that is generated by the helicopter rotor under different operating conditions.

Together with the flow patterns, Fig. 6 also shows the characteristic shapes of the brownout clouds that are produced by the rotor when operated in each of the modes just described. The diagrams suggest that, if the pilots were to dwell for too long in the recirculatory mode (Fig. 6b), then this would pose the greatest threat to the helicopter, leading rapidly as it might to a dense region of rapidly-moving dust forming very close to where the cockpit of the helicopter is likely to be. In contrast, the possibility exists in hover (Fig 6a) that the regions of the cloud where the dust is most dense might be forced back away from the aircraft, reducing the danger that is posed by the development of the dust cloud. Once the aircraft is flown fast enough for the flow to enter the ground vortex mode (Fig 6c),

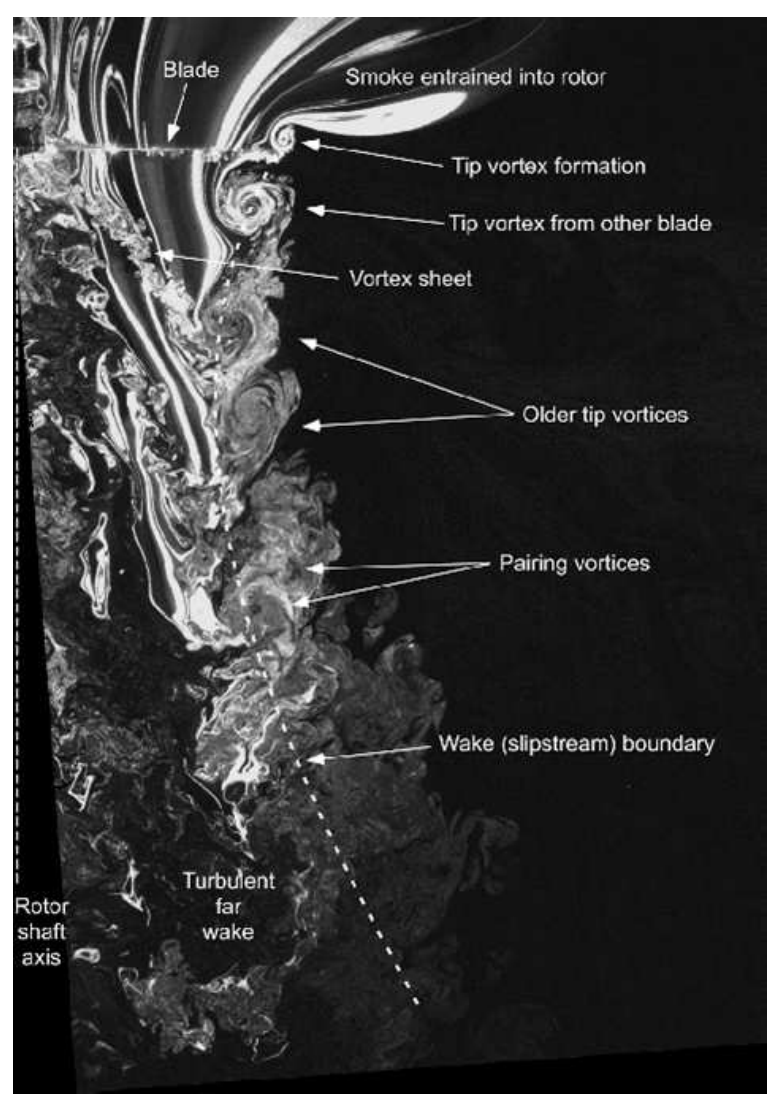

Figure 8: The structure of the flow field that is produced by a small model rotor in hover in free air, as visualised by injecting smoke into the flow just above the rotor. (Image from Ref. 5 with permission of the authors.)

the dust cloud would be swept back and behind the aircraft where, in most instances, it would likely do little to obscure the pilots' view of the ground.

These patterns are modified somewhat by the details of the helicopter geometry. For instance, the flow field that is produced by a tandem-rotor helicopter is characterised by strong interaction between the individual flow fields that are generated by the front and rear rotors. Nevertheless, Fig. 7 shows that the structure of the dust clouds that are generated by the tandem-rotor helicopter when operating in each of the flow modes still retains the essential features of the dust clouds that are produced by the single-rotor system.

The extreme generality of the observations that have just been presented is a consequence of the simplicity of the model that was used to generate them, and the superficiality of this analysis should be borne in mind when considering its applicability to specific aircraft types. It is important also to note that the history of the flow can have a profound effect on the geometry of the cloud, and this is not easily accounted for when using this simple model. For instance, if the aircraft were to manoeuvre close to the ground, this would result in the cloud passing through a succession of states, each of which would retain some imprint of the earlier history of the aircraft's dynamics. This obscures the 
association of a particular form of dust cloud with each individual flow mode that can be produced by the rotors of the helicopter in the presence of the ground.

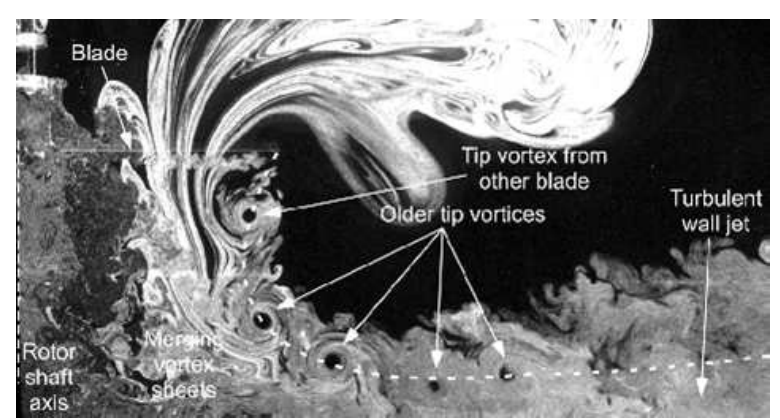

Figure 9: The structure of the flow field that is produced by a small model rotor in hover above a ground plane, as visualised by injecting smoke into the flow just above the rotor. (Image from Ref. 5 with permission of the authors.)

The simple description of the fluid dynamics of the rotor that was used to inform this rather crude picture of the shape and size of the brownout cloud can be augmented, however. Figure 8 shows the structure of the wake of a small model rotor in hover in free air, as visualised by injecting smoke into the flow just above the rotor. The downwash through the rotor is

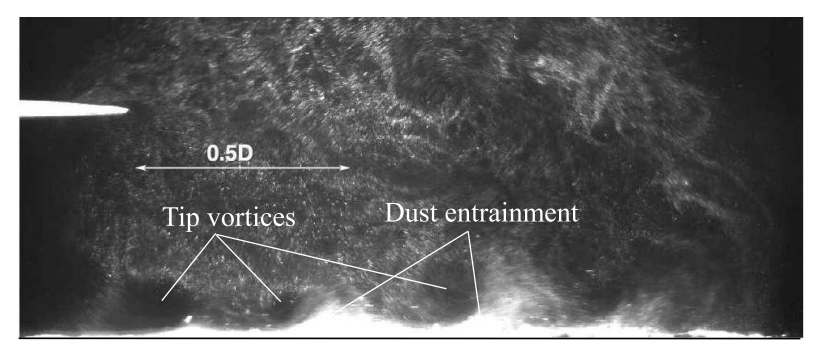

Figure 10: Wind-tunnel visualisation of dust entrainment from the ground into the flow surrounding a small model rotor in slow forward flight. The rotor is the bright object at top left, and the air-flow in the wind tunnel is from right to left. (Image from Ref. 4 with permission of the authors.)

in fact induced by a sequence of helical vortices that emanate from the blade tips. These vortices travel downwards into the flow below the rotor. As they do so, they interact in unstable fashion so that, by about two radii below the rotor, the regular structure of the vortices within the rotor wake has degenerated into a disordered tangle of interwoven vortex filaments. The role of this vortical instability in moderating the sensitivity of the system to changes in the rotor design will be discussed later in this paper. Figure 9 shows the flow produced by the same rotor when hovering close to the ground. It can be seen clearly how the individual vortices that are produced by the rotor blades pass very close to the ground before interacting with each other and eventually entangling to produce a dis-
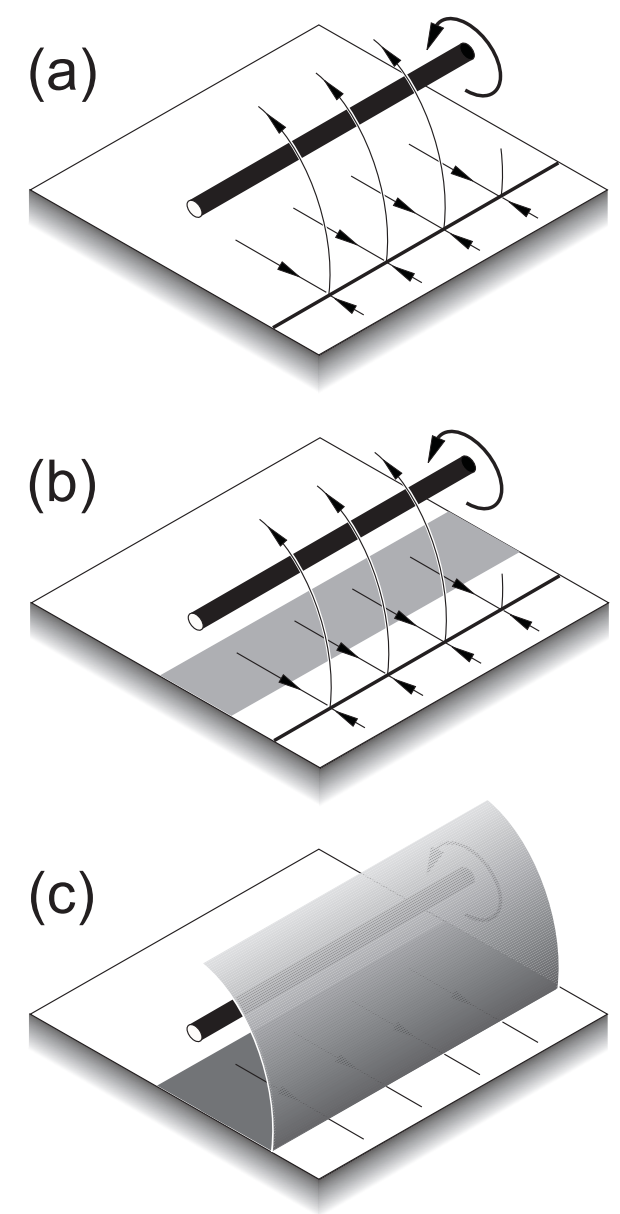

Figure 11: Schematic showing how an extended vortex, moving parallel to the ground, may produce a sheet-like distribution of dust in the flow. Part (a) shows the flow trajectories associated with the vortex, (b) the location of the particulate source on the ground (dark patch) and (c) the resultant dust distribution.

ordered, turbulence-like flow some distance outboard of the rotor. Careful experimental visualisations of the behaviour of the system when seeded with a layer of particulates on the ground below the rotor show the scouring effect of these vortices as they pass just above the ground plane to be fundamental in inducing particulate matter to leave the ground and enter the flow (see Fig. 10).

Indeed, the existence of these vortex-ground interactions is fundamental in explaining the preponderance of sheet- and filament-like structures within the brownout cloud (such as those shown in Fig. 5). In the light of the model of the entrainment process described earlier, it can be imagined how the close passage of one of these vortices, by producing a region of locallyelevated velocity on the ground below, will activate the source of particulates within a strip-like patch on the ground (see Fig. 11). The topology of the flow created by the vortex then plays an essential role in initiating the formation of the brownout cloud. More specifically, the separation line that forms just upstream of 
the vortex provides a route through which the particulates can be transported away from the ground and into the flow. Without this feature, the particulates would remain in a thin layer just above the ground and would thus not contribute to the formation of the dust cloud. Careful interrogation of detailed simulations show that vortices that lie essentially parallel to the ground are responsible for sheet-like features in the dust cloud, while vortices that have become distorted or tangled by their interaction with other vortical structures in the flow tend to be responsible for the filament-like structures that are often also observed to be present (Ref. 15). These observations lead to the fundamental insight that if the structure and strength of these individual vortices could be changed, then so might also the properties of the dust cloud that is produced by the rotor under brownout conditions.

\section{Effect of Rotor Design}

Anecdotal evidence suggests that different types of helicopter have distinctly different behaviour under brownout conditions. Although still somewhat controversial, such evidence suggests that various aspects of the aerodynamic design of its rotor may have an influence on the size and shape of the dust cloud that is produced by a helicopter under brownout conditions. The most detailed numerical models are capable of capturing these effects. Figures 12 and 13 show the mean particulate density that is predicted to form on a vertical slice through the longitudinal centreline of a five-bladed rotor when operating in ground vortex mode. To produce these predictions, the authors' high-resolution computational model for the coupled dynamics of the air and the dust particles suspended therein, called the VTM/PTM, was used (Ref. 10). In Fig. 12, the dust density distribution produced by the rotor is compared for various practical values of the twist of the rotor blades, with the rotor operating at the same thrust coefficient in all cases. It is clearly evident that the model predicts the size and density of the dust cloud surrounding the helicopter to have a distinct sensitivity to blade twist, with the rotor having blades with $11^{\circ}$ twist yielding, at least qualitatively, the most diffuse dust distribution (see Ref. 11 for a discussion of the effect of varying some of the other parameters of the rotor design, and Ref. 1 for a discussion of the effect of helicopter configuration on the properties of the resultant dust cloud). Figure 13 compares similar data but where the tip shape of the blades instead of their twist has been varied. All tip shapes appear to result in almost identical distributions of dust in the airflow surrounding the rotor except, curiously, the BERP rotor which seems to produce a greater preponderance of dust in the ground vortex than the blades with other tip geometries.
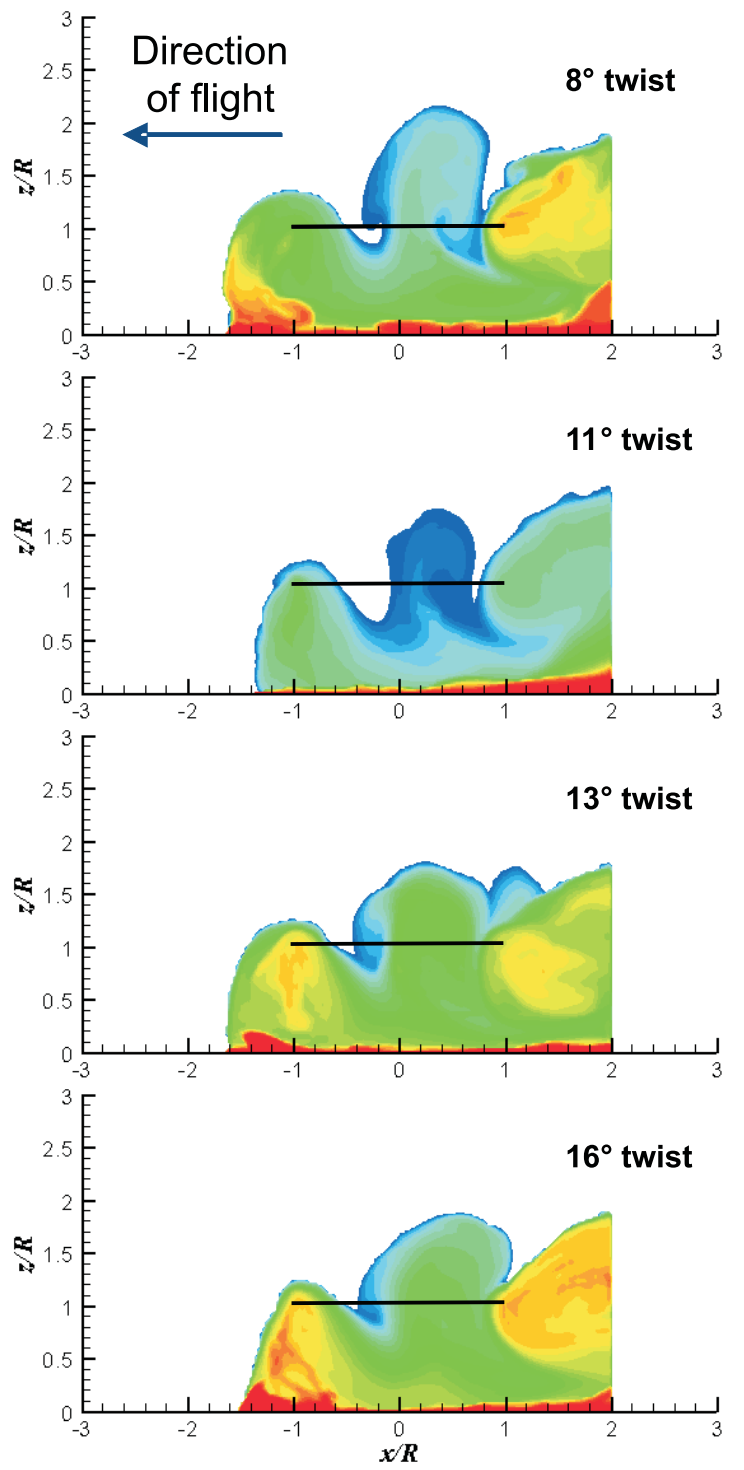

Figure 12: Sensitivity to blade twist of the dust density in the flow surrounding a five-bladed rotor in slow forward flight. (As visualised on the vertical plane through the longitudinal centreline of the rotor. Red denotes the highest dust density, blue the lowest.)

In practical engineering terms it is obviously much more feasible to implement relatively superficial changes to the rotor geometry rather than major alterations to its design. For instance the tip shape of the blades can be changed more readily than the twist or any of the other more fundamental parameters of the rotor. The curious feature of the simulations just presented is that, although it is known that both the twist and the tip shape can and do have a significant effect on the loading on those parts of the blade which are crucial to the formation and subsequent evolution of the tip vortex, the effect of the tip does not appear to propagate through the system to yield appreciable effect on the shape, size or density of the dust cloud whereas the twist distribution does. The question thus arises as to why the brownout cloud might not be as 

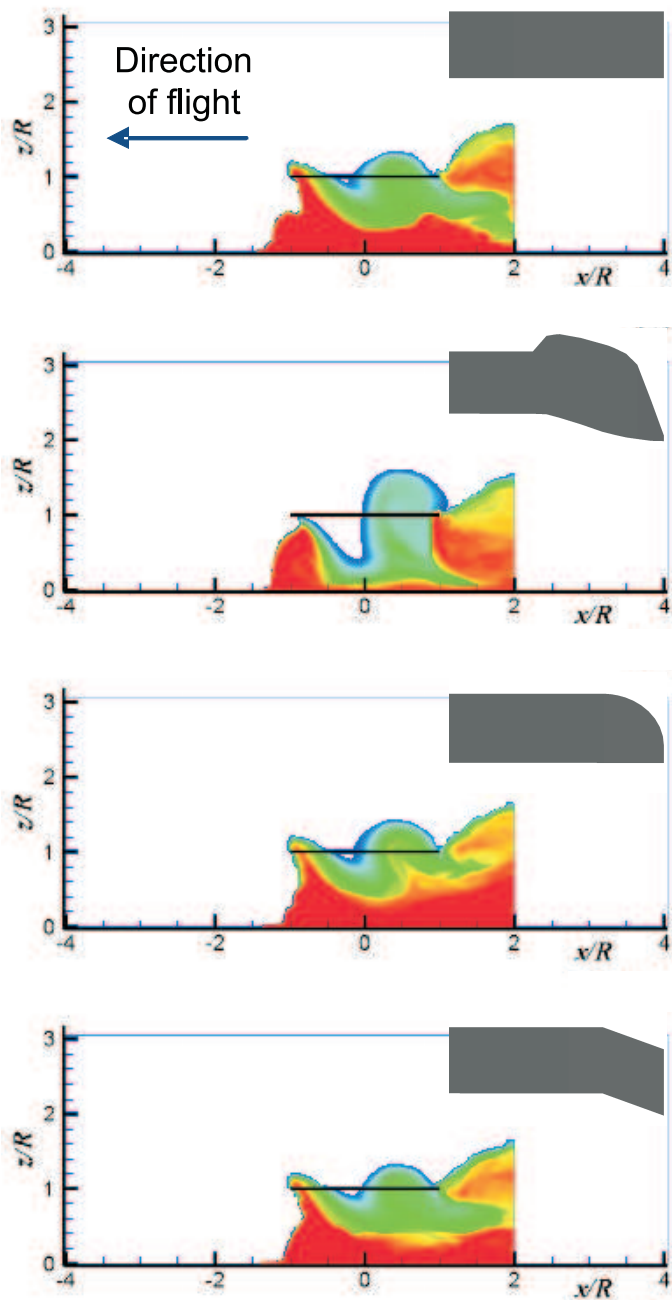

Figure 13: Sensitivity to blade tip shape of the dust density in the flow surrounding a five-bladed rotor in slow forward flight. (As visualised on the vertical plane through the longitudinal centreline of the rotor. Red denotes the highest dust density, blue the lowest.)

readily influenced by some of the possible changes to the rotor geometry as others, even though all might be known to have a strong and direct effect on the flow structures that are important to the generation of the dust cloud.

Although the detailed dependence of the shape, size and density of the dust cloud on the rotor design cannot be inferred from the approximate models described earlier, these models can be used to guide the interrogation of more complex and complete simulations of the development of the brownout cloud. It seems reasonable to accept the insight from the simplest approximations that the gross shape and size of the dust cloud is sensitive to the details of the rotor design only to the extent that the gross characteristics of the flow pattern that is produced by the rotor are. Then, in order to understand the (in)sensitivity of the dust cloud to the details of the rotor design, one is led to focus on the manner in which the tip vortices interact with the ground in order to entrain particulate matter into the flow in the first instance. Figure 14 shows a snapshot of the vortical structure of the wake at one particular instant in a simulation of a hovering rotor that was conducted using the VTM/PTM model. Figure 14 also shows the distribution of the active regions of entrainment of sediment from the ground into the flow at the same instant. In other, similar pairs of snapshots taken of the flow at earlier or later times, the observed patterns are qualitatively similar to those shown, but are often quite radically different in their detail.

It is very clear from these images that the regions of maximal uplift of dust from the ground into the flow are very closely correlated with the locations of the individual vortical structures that are present in the flow near to the ground plane. This is to be expected from the predictions of the simple structural model presented earlier in this paper.

More interestingly, however, two distinct regimes can be identified. Close to the rotor, the sediment is entrained into the flow in a series of concentric waves, and these waves propagate outboard along with their associated tip vortices. Further outboard, however, the pattern of entrainment becomes increasingly more disorganised, so that, at about two radii outboard from the rotor, the wave-like features found further inboard are replaced almost entirely by a highly granular and disordered pattern of localised sediment uplift with characteristic length-scales that are much smaller than the rotor radius.

\section{Fundamental Role of Vortex Instability}

This transition from orderly entrainment close to the rotor into disorder at some distance away from the rotor is associated with the natural instability of the vortex filaments that constitute the wake of the rotor. The progression of this instability is clearly evident in Fig. 14 which shows the helical structure of the younger elements of the vortices close to the rotor soon to be perturbed by the mutual induction of neighbouring filaments so that a short distance outboard of the periphery of the rotor, their mutual interaction leads to such intense stretching and re-orientation that all helical symmetry in the vortices, and a good deal of their coherence, is lost.

In terms of its influence on the development of the brownout cloud, the development of this instability results in a competition between two different physical effects within the flow. The imprint of the details of the rotor design is left on the distribution of vorticity that is generated behind the rotor blades. This is a basic consequence of the effect that the design of the 

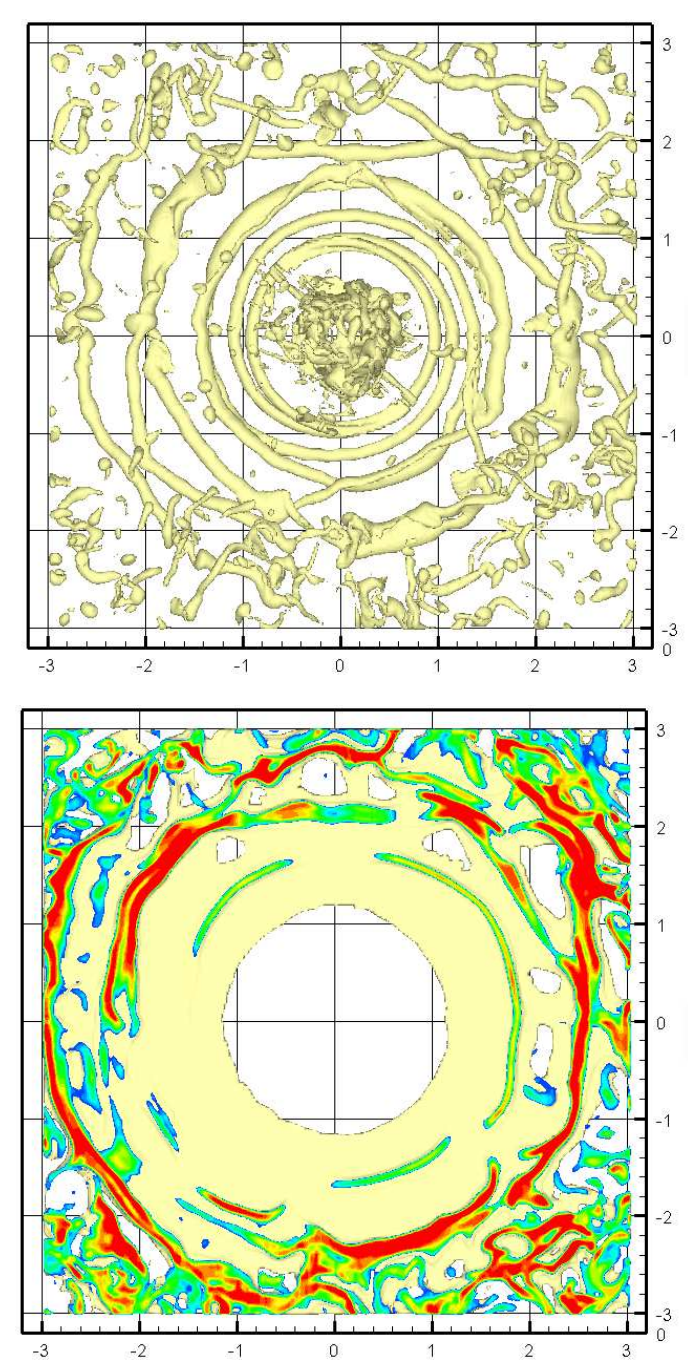

Figure 14: Snapshot comparing the vorticity distribution in the flow (top) to the regions of maximal particulate uplift from the ground (bottom: red = highest, blue = lowest) for a two-bladed rotor in hover at half a rotor radius above the ground (Ref. 15).

blade has on the distribution of lift along its length. The action of the vortical instability in disordering the wake is then to wash out or diffuse this imprint over time (see Fig. 15). Thus only those changes to the rotor design that imprint on the wake structure in robust enough fashion to persist all the way from the rotor to the ground plane in the presence of this instability will yield an effective means of modifying the brownout cloud that is produced by the rotor under practical conditions.

The important question thus remains as to how we might react to overcome this natural insensitivity of the physics of the evolution of the dust cloud to changes to the rotor design, in order to create a rotor that has more favourable brownout characteristics than those currently in use.

One of the major impediments to rapid progress in this vein is that, although the behaviour of rotor wakes in free air has been studied relatively intensely, very little is actually known in detail about how the natural instability of the rotor wake develops in the presence of the ground. Nevertheless, it is known that the growth rate of the vortical instability is exponential. It will thus be very difficult, perhaps even impossible, to implement any mitigation strategy that relies on stabilising the flow, or, indeed, on the flow being inherently stable in the first place.

On the other hand, it may be possible to de-stabilise the vortical system with positive effect on the characteristics of the dust cloud. Initial work by Leishman with slotted blade tips (Ref. 16) has shown promising results in terms of promoting the instability of the individual vortices within the wake (as distinct from the overall vortical structure of the wake as alluded to above) and the obvious next step in his research will surely be to explore the performance of this tip in the context of brownout amelioration. The new tip introduces a performance penalty, at model scale, of $2-3 \%$ in power required to hover. This may not be acceptable in practice, but further optimisation holds significant promise.

Some of our calculations have hinted too (Ref. 17) that it might indeed be possible to de-stabilise the entire wake to produce a flow that is very similar to that encountered in the vortex ring state. When the rotor wake is induced to collapse into this form, individual tip vortices never approach the ground sufficiently closely to activate the source of particulates on the surface and the rotor becomes essentially immune to brownout. A significant performance penalty is currently incurred with this approach, however, and additional research is ongoing in order to eliminate this.

\section{Conclusion}

It is hoped that the reader is convinced by what has been written here that the answer to question posed in title to this paper is an emphatic 'yes' and indeed that modelling efforts, although by no means having run their full course to maturity, have advanced significantly our understanding of the physics that underpins the formation and development of the dust cloud that can be induced to form in the air surrounding a rotorcraft under brownout conditions. If anything, the last few years of intense activity have led researchers to adopt a less naive view of the challenges ahead in ameliorating the effects of brownout, whether through aerodynamic changes to the helicopter or through more active intervention. There is certainly no quick fix to the problem. Aerodynamic amelioration will be difficult and will require a much deeper and more subtle understanding of the basic physics of brownout - particularly of how sediment leaves the ground and enters the flow around the helicopter in the first place. Progress is being made 
on these difficult issues, and we are just beginning to understand the balance between rotor design and the natural tendency of the aerodynamics of the system to suppress the effects of changes to the design on the dynamics of the brownout cloud. Nevertheless it is the authors' opinion that the pay-offs of aerodynamic rather than systems-based amelioration will be greater in the long term and will better address the trade-offs on the performance of the system that will most likely accompany any practical ameliorative strategy. The concessions on performance that will result from implementing suitable modifications to the rotor blades can in many cases be circumvented operationally, or so we believe, and such an approach is largely preferable to simply throwing payload away by carrying kilogrammes of highly-specialised and rarelyused electronic equipment on board the aircraft.

The full benefits of academic participation in brownout-related research will not be achieved however without a significant change in the short-term attitude that seems to be prevalent (albeit with some justification) amongst those who have the power to promote and direct research in this important field. History lends the rather gloomy projection that this will not be the last time that a major war will be fought under conditions similar to those which have stimulated present interest in the physics of helicopter brownout. History also motivates us to consider whether or not we will be better prepared for events next time.

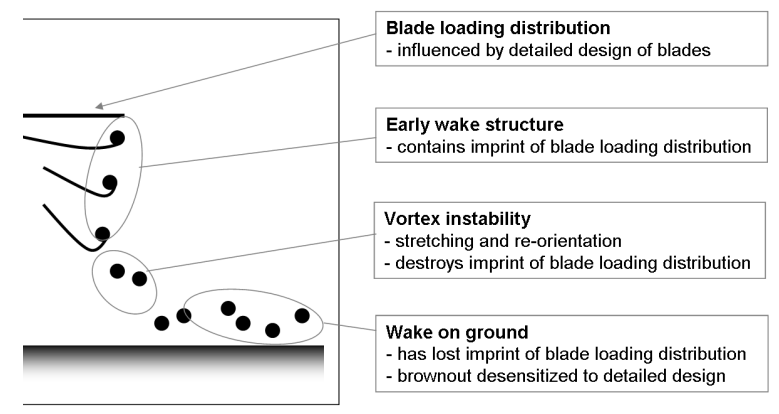

Figure 15: Schematic that summarises the proposed link between blade loading and the formation of the brownout cloud, as moderated by the natural instability within the rotor wake.

\section{Acknowledgements}

The authors would like to acknowledge the fruitful discussions with J. Gordon Leishman and F. John Perry during the research leading to this paper. The opinions expressed within this paper remain those of the authors, however.

\section{References}

${ }^{1}$ Phillips, C. and Brown, R.E., "The Effect of Helicopter Configuration on the Fluid Dynamics of Brownout," 34th European Rotorcraft Forum, Liverpool, UK, 16-19 September 2008.

${ }^{2}$ Rodgers, S.J., "Evaluation of the Dust Cloud Generated by Helicopter Rotor Downwash," USAAVLABS Technical Report 67-81, U. S. Army Aviation Material Laboratories, 1968.

${ }^{3}$ Cowherd, C., "Sandblaster 2 Support of See-Through Technologies for Particulate Brownout," MRI Project No. 110565.1.001, Midwest Research Institute, 2007

${ }^{4}$ Nathan, N.D., and Green R.B., "Measurements of a Rotor Flow in Ground Effect and Visualisation of the Brownout Phenomenon," American Helicopter Society 64th Annual Forum Proceedings, Montreal, Canada, 29 April1 May, 2008.

${ }^{5}$ Johnson, B., Leishman, J.G., and Sydney, A., "Investigation of Sediment Entrainment in Brownout Using High-Speed Particle Image Velocimetry," 65th Annual Forum of the American Helicopter Society, Grapevine, TX, 27-29 May 2009.

${ }^{6}$ Syal, M., Govindarajan, B., and Leishman, J.G., "Mesoscale Sediment Tracking Methodology to Analyze Brownout Cloud Developments," 66th Annual Forum of the American Helicopter Society, Phoenix, AZ, 11-13 May 2010

${ }^{7}$ Wachspress, D.A., Whitehouse, G.R., Keller, J.D., McClure, K, Gilmore, P., and Dorsett, M., "Physics Based Modeling of Helicopter Brownout for Piloted Simulation Applications," Interservice/Industry Training, Simulation and Education Conference (I/ITSEC), Orlando, Florida, Dec 2008.

${ }^{8}$ Wachspress, D.A., Whitehouse, G.R., Keller, J.D., K., Yu, Gilmore, P., Dorsett, M., and McClure, K, "A High Fidelity Brownout Model for Real-Time Flight Simulations and Trainers," 65 th Annual Forum of the American Helicopter Society, Grapevine, TX, 27-29 May 2009.

${ }^{9}$ DAndrea, A., "Numerical Analysis of Unsteady Vortical Flows Generated by a Rotorcraft Operating on Ground: a First Assessment of Helicopter Brownout," 65th Annual Forum of the American Helicopter Society, Grapevine, TX, 27-29 May 2009.

${ }^{10}$ Phillips, C. and Brown, R.E., "Eulerian Simulation of the Fluid Dynamics of Helicopter Brownout," Journal of Aircraft, Vol. 46 (4), July 2009, pp. 1416-1429.

${ }^{11}$ Phillips, C., Kim, H.W., and Brown, R.E., "The Effect of Rotor Design on the Fluid Dynamics of Helicopter Brownout," 35th European Rotorcraft Forum, Hamburg, Germany, 22-25 September 2009.

${ }^{12}$ Munro, R.J., Bethke, N., and Dalziel, S.B., "Sediment Resuspension and Erosion by Vortex Rings," Physics of Fluids, Vol 21 2009, pp 046601:1-16.

${ }^{13}$ Curtiss, H.C. Jr., Sun, M., Putman, W.F., and Hanker, E.J. Jr., "Rotor Aerodynamics in Ground Effect at Low Advance Ratios," Journal of the American Helicopter Society, Vol. 29 (1), 1984, pp. 4855 .

${ }^{14}$ Whitehouse, G.R. and Brown, R.E., "Modelling Rotor Wakes in Ground Effect," Journal of the American Helicopter Society, Vol. 49 (3), July 2004, pp. 238-249.

${ }^{15}$ Phillips, C., Kim, H.W., and Brown, R.E., "The Flow Physics of Helicopter Brownout," American Helicopter Society 66th Annual Forum Proceedings, Phoenix, AZ, 11-13 May, 2010.

${ }^{16}$ Milluzzo, J., Sydney, A., Rauleder, J., and Leishman, J.G. "In-Ground-Effect Aerodynamics of Rotors with Different Blade Tips," American Helicopter Society 66th Annual Forum Proceedings, Phoenix, AZ, 11-13 May, 2010.

${ }^{17}$ Phillips, C.. "Computational Study of Rotorcraft Aerodynamics in Ground Effect and Brownout," PhD dissertation, University of Glasgow, Glasgow, Scotland, January 2010. 